\title{
Prevalence of vascular complications and factors predictive of their development in young adults with type 1 diabetes: systematic literature review
}

Steven James ${ }^{1 *}$, Robyn Gallagher ${ }^{2}$, Janet Dunbabin ${ }^{3}$ and Lin Perry ${ }^{4}$

\begin{abstract}
Background: Vascular complications curtail life expectancy and quality of life in type 1 diabetes and development at younger ages is particularly detrimental. To date no review has summarised the prevalence or factors predicting their development in young adults.

Methods: A quantitative epidemiological systematic review was conducted to identify the prevalence and predictive factors for development of retinopathy, nephropathy and hypertension in young adults (sample age mean [plus 1SD] 18-30 years) with type 1 diabetes, using processes adapted from established review methods set out by the Centre for Reviews and Dissemination.

MEDLINE (Ovid), Scopus (Elsevier), CINAHL, Science Direct (Elsevier), Google Scholar and Cochrane databases were searched to identify relevant articles published between 1993 and June 2014. From this eleven papers were retrieved, appraised and results summarised by three reviewers using established methods.

Results: Some form of retinopathy occurred in up to almost half of participants; more severe forms affected up to one in ten. One in six was reported with microalbuminuria; one in 14 had macroalbuminuria. Hypertension occurred in almost one in two participants. Applying out-dated high thresholds this decreased to approximately one in ten participants. Glycaemic control was a consistent predictor of vascular disease in this age group.

Conclusion: Prevalence rates of retinopathy, nephropathy and hypertension in young adults with type 1 diabetes emphasise the importance of regular complication screening for early detection and treatment. The predictive effect of glycaemic control reinforces its importance for prevention of vascular complications.
\end{abstract}

Keywords: Quantitative epidemiological systematic review, Vascular complications, Prevalence, Predictors, Retinopathy, Nephropathy, Hypertension, Young adults, Type 1 diabetes

\section{Background}

The increasing incidence of type 1 diabetes in many countries challenges health systems because the disease is presently incurable with no known method of prevention [1]. Around 490,100 children live with the disease worldwide, with incidence estimated to be increasing in children under 15 years by $2.8 \%$ per year $[1,2]$. This trend is particularly worrying because type 1 diabetes increases mortality and morbidity population-wide, including in young adults [3-5]. People with type 1 diabetes

\footnotetext{
* Correspondence: Steven.B.James-1@student.uts.edu.au

${ }^{1}$ Huntsville District Memorial Hospital, Muskoka Algonquin Healthcare, 100

Frank Miller Drive, Huntsville, Ontario P1H 1H7, Canada

Full list of author information is available at the end of the article
}

diagnosed before the age of 30 years have been calculated to have a 4.7-fold excess mortality risk [6].

Vascular complications are often the cause of this early mortality. However, there is currently little information available about the prevalence or predictive factors for development of vascular complications of type 1 diabetes among young adults. Identification of disease complication prevalence and any predictive characteristics will establish a benchmark of these risk factors, and may assist healthcare professionals to target appropriate information and support with the aim of deferring or averting their onset.

The development of vascular disease in type 1 diabetes has been proposed as a consequence of disordered activity 
of lipid metabolism enzymes or transporters affecting endothelial function, inflammation, coagulation, platelet activation and fibrinolysis [7]. In combination with populationwide cardiovascular and athero-thrombosis risk factors, a state of persistent and progressive damage to the vascular wall (macro-angiopathy) is created [8]. Micro-angiopathic disease also occurs, with hyperglycaemia a leading pathogenic factor [9]. Vascular co-morbid diseases include retinopathy, which may cause reduced vision and blindness, and nephropathy, which may result in renal failure and require dialysis or kidney transplantation. This is in addition to hypertension, which is linked to peripheral, cardio- and cerebrovascular disease, the end points of which are limb amputations, cardiac failure, stroke and sudden death. As vascular complications curtail both life expectancy and quality of life [10], development at younger ages when people are typically establishing careers and families is particularly detrimental.

Young adults may be particularly vulnerable to complications because many have unique health needs relating to their psychological, physical and socio-cultural life stage issues; these commonly lie outside health services' remit and place them at high risk for poor self-management. Diabetes services are predominantly structured into exclusive paediatric and adult services, with transition between these services not clearly the responsibility of either: an arrangement which serves young adults poorly. This transition stage was first formally identified as challenging by Blum et al. [11], however, decades later similar levels of difficulty with the transition process are still being reported [12]. Consequently, young adults with type 1 diabetes may not adhere to diabetes regimes, and may disengage from diabetes services after transition [13,14]. Attrition from, or failure to engage with, diabetes services as an adult toofrequently results in reduced diabetes self-management and well-being, and inadequate screening for complications.

The potential benefits of models of transition to maintain engagement with adult diabetes services post-transfer from paediatric care have been flagged, and characteristics associated with reduced attrition and increased satisfaction and successful service redesign described [15-17]. However, the effect of transition service redesign has not been examined in terms of outcomes such as onset of vascular complications; neither has there been any attempt to summate or quantify the degree of complication-related morbidity and early mortality experienced by this young adult group. Lack of international benchmarks limits evaluation and deters appropriate prioritisation of service redesign to promote retention of young adults in contact with services, an essential element in achieving good glycaemic control to defer onset of complications [18-20].

The aim of this review was to identify the prevalence and factors predictive of development of vascular complications (retinopathy, nephropathy and hypertension) occurring in young adults with type 1 diabetes. For the purpose of this review, the term young adult refers to ages 18-30 years inclusive.

\section{Methods}

A quantitative epidemiological systematic review was conducted using processes adapted from established review methods set out by the Centre for Reviews and Dissemination [21]. Standards derived from the Preferred Reporting Items for Systematic Reviews and Meta-Analysis (PRISMA) were applied [22]. The review protocol is available from the authors on request.

\section{Outcome definitions and recommended measurement methods}

Definitions and criteria for 'best practice' screening methods for retinopathy, nephropathy and hypertension were sought. Detailed recommendations were available in American, Canadian and British guidelines [23-25]:

\section{Diabetic retinopathy}

The presence and characteristic evolution of typical retinal microvascular lesions in an individual with diabetes. Besides micro-aneurysms, blood vessel changes include intra-retinal haemorrhage, and vascular tortuosity and malformation (non-proliferative retinopathy) leading to abnormal vessel development (proliferative retinopathy). Seven-standard field stereoscopic-colour fundus photography with interpretation by a trained reader is the recommended standard screening for diabetic retinopathy, though direct ophthalmoscopy or indirect slit-lamp fundoscopy through dilated pupil or digital fundus photography may also be used. Treatment with laser photocoagulation surgery prevents vision loss [26-29]. The Canadian Diabetes Association Clinical Practice Guidelines Expert Committee [24] advocates that screening should be undertaken at least annually. However, the American Diabetes Association [23] advocates consideration of lesser frequency (every two - three years) following one or more normal eye examinations.

\section{Nephropathy}

A glomerular filtration rate (GFR) less than $60 \mathrm{~mL} / \mathrm{min}$ present for three or more months, or any evidence of kidney damage for three or more months regardless of GFR [30]. In addition to any anatomical or pathological abnormalities or glomerular haematuria, it can be revealed by micro- or macroalbuminuria/proteinuria. Screening for nephropathy in adults with diabetes entails estimation of the level of kidney function and assessment of urinary albumin excretion. Significantly reduced kidney function is evidenced by an estimated GFR less than $60 \mathrm{~mL} / \mathrm{min}$; serum creatinine should be used to estimate GFR and stage the level of chronic kidney disease. Albuminuria should be 
determined through a timed/24-hour collection, or through a random spot test to determine albumin to creatinine ratio (ACR). The measurement of a spot urine for albumin, without simultaneously measuring urine creatinine, is susceptible to false negative/positive determinations.

Microalbuminuria was identified as urinary albumin excretion of either 30-299 or $300 \mathrm{mg} /$ day in a 24-hour urine collection, with variations based on differing guidelines [23,24], or an ACR of $2.0-20.0 \mathrm{mg} / \mathrm{mmol}$. Macroalbuminuria (overt nephropathy) was identified as $300 \mathrm{mg} /$ day or above if a 24-hour urine collection was performed, or an ACR of greater than $20.0 \mathrm{mg} / \mathrm{mmol}$.

\section{Blood pressure}

Recommended targets are less than 130/80 $\mathrm{mmHg}$ for people with diabetes. Measurement of blood pressure should be undertaken by trained personnel, with participants in the seated position with feet on the floor and arm supported at heart level, after five minutes of rest. Cuff size must be appropriate for the arm circumference, with elevated values confirmed on a separate day. The American Diabetes Association [23] advocate that blood pressure should be measured at every routine visit.

\section{Literature search methods}

MEDLINE (Ovid) and Scopus (which incorporates Embase journals), CINAHL, Science Direct (Elsevier), Google Scholar and Cochrane were searched by the first author to June 2014 to identify relevant articles. The MESH headings 'Diabetes Mellitus, Type 1'; 'Diabetic Retinopathy'; 'Diabetic Nephropathies'; 'Hypertension'; 'Prevalence'; 'Cross-sectional Studies'; and 'Prospective Studies', and keywords 'Type 1 diabetes'; 'Insulin Dependent Diabetes Mellitus'; 'Juvenile Onset Diabetes Mellitus'; 'Retinopathy'; 'Eye Diseases'; 'Nephropathy'; 'Kidney Diseases'; 'High Blood Pressure'; and Longitudinal Studies' were used. The full search strategy can be viewed in Additional file 1. In addition, reference lists of all eligible studies were handsearched.

Inclusion criteria were:

- Samples with type 1 diabetes;

- Mean age (plus 1SD) 18-30 years, or where the results for this age range were reported separately from other age groups; and

- English language studies only due to lack of resources for translation.

\section{Exclusion criteria:}

- Studies reporting data collected pre 1993 as from this date the definitive Diabetes Control and Complications Trial [9] established that the onset and progression of micro-vascular complications can be significantly reduced by HbA1c management. This changed diabetes management to make glycaemic control central, and hence management and complication rates may have changed.

\section{Search outcome}

A total of 7,740 records were identified, downloaded to EndNote version $\mathrm{X} 4$ and screened by reading titles and abstracts. Of these, 7,601 records were excluded as duplicates or not meeting review inclusion criteria, including 12 non-English language papers. The remaining 139 full-text articles were assessed for eligibility; their reference lists were searched and an additional 12 papers identified. Of these 151 papers, 140 did not meet review inclusion criteria, leaving eleven relevant papers [31-41]. The search process and outcomes are summarised in Figure 1.

\section{Quality appraisal}

With no universally accepted 'gold standard' method for evaluating and interpreting epidemiological study quality [42], to determine the strength of evidence quality appraisal was undertaken using the Strengthening the Reporting of Observational Studies in Epidemiology (STROBE) [43] checklist for cohort, case-control, and cross-sectional studies. Eligible papers were also evaluated for methods of assessment and measurement of retinopathy, nephropathy and hypertension in relation to current evidence-based guideline recommendations. This appraisal can be viewed in Additional file 2. To ensure reliability in data extraction and quality appraisal, a sample of papers included in the review were independently appraised and data extraction compared by the second and last authors (six papers each). Agreement was reached for all papers.

\section{Data extraction and synthesis}

Data were extracted to a purpose-designed spread-sheet in Microsoft Office Excel based on relevant elements of the Consolidated Standards of Reporting Trials (CONSORT) checklist [44]. Extracted data can be viewed in Table 1 and Additional file 3. The number of diabetes centres involved in each study was noted to aid interpretation of transferability of findings.

\section{Results}

The eleven papers derived from nine separate studies and mainly employed cross-sectional research designs; three papers that had provided data applicable to the target age group had involved a 1995 cohort of a Danish nationwide longitudinal study $[32,37,38]$. Data were collected via case note audit in three studies, and via documentation surveys in two further studies. Only three of the eleven studies solely provided data relating to the 


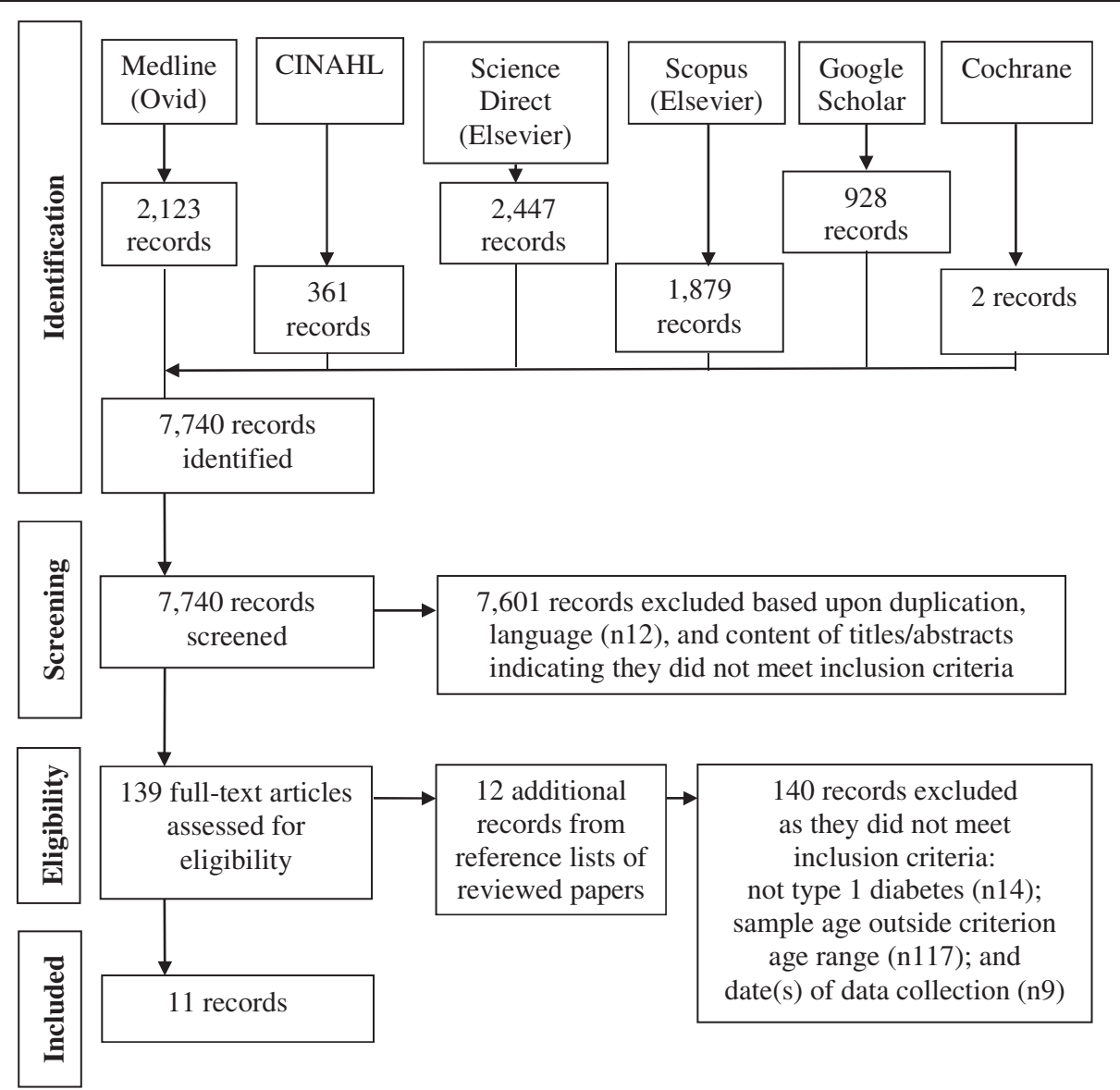

Figure 1 Literature search and review flow chart.

target population; all varied in their methodological quality and are summarised in Table 1 and Additional files 2 and 3 . Ethnicity was reported in only three papers, and only one study focused on rural/non-urban populations or localities.

\section{Prevalence and prediction of retinopathy}

The prevalence of diabetic retinopathy in the target population was reported in seven papers [31,32,34,36-38,40]. Prevalence data were reported for 215 applicable participants by Arfken et al. [31], for both 95 (2010) and 85 (2011) participants by James et al. [34], and 53 by Salardi et al. [40]. In longitudinal studies data were also reported on 324 applicable participants by Broe et al. [32], on 290 at nine years diabetes duration by LeCaire et al. [36], on 190 by Olsen et al. [37], and on 353 by Olsen et al. [38]. These two latter papers reported data from 19 paediatric departments (both) and five/six departments of internal medicine, whereas Salardi et al. [40] reported data from eleven centres; the other four papers did not provide detail.

Retinopathy was assessed and measured according to current best practice guideline recommendations in all papers. In these seven studies from four different countries with participants sampled by different methods, retinopathy prevalence varied somewhat (Table 1). Salardi et al. [40] reported an overall prevalence of $40 \%$, with $27 \%$ and $88 \%$ at less than or greater than 20 years diabetes duration, respectively, whereas James et al. [34] reported a prevalence of $13.7 \%$ and 9.4\%, and Olsen et al. [38] 57.6\%; LeCaire et al. [36] reported $47 \%$ with retinopathy at nine years diabetes duration $(6 \%-73 \%$ with retinopathy at mean ages 19.5 - 24.8 years). At similar diabetes duration proliferative or treated diabetic retinopathy was reported in $10.2 \%$ of participants by Arfken et al. [31], but affected 0.3\% of those at nine years diabetes duration by LeCaire et al. [36]; Broe et al. [32] reported a prevalence of proliferative retinopathy of $0.5 \%-0.7 \%$.

Data were provided relating to predictors of diabetic retinopathy in the target population in only two of these studies. Arfken et al. [31] reported that in White participants, a strong association was demonstrated between the development of proliferative retinopathy and existing moderate/severe diabetic retinopathy (Odds Ratio (OR) 16.55 (95\% Confidence Interval (CI) 5.43 - 50.45)). Glycaemic control was also shown to be significant (2\% change in HbA1c; OR 2.17 (95\% CI 1.34 - 3.50)). This 
Table 1 Reported prevalence of diabetic retinopathy

Author(s); Number Age, mean (SD) yrs.; sample size

country of 


\section{Olsen et al. \\ 19 and \\ Median 21.1 (range 12.0 - 26.9); n 205 > 20 yrs. (n 339 TS)}

1999; Denmark five $\wedge$

\section{[37]}

Olsen et al. $\quad 19$ and

2004; Denmark six

[38]

20.4 (3.2) (Prepubertal diabetes onset), 24.2 (1.3) (Pubertal/post-pubertal 57.6\%

diabetes onset); $n 304$ (Prepubertal diabetes onset), n 49 (Pubertal/post-

pubertal diabetes onset ) (n 353 TS)

\section{Salardi et al.}

2012; Italy [40]
48.9\% (Min)

20\% (Mod

plus)

HbA1c (DR); $p<0.0001$

Diabetes duration

before puberty (DR);

$p<0.05$

after the onset of puberty

(DR); $p<0.001$

(DR) Diabetic retinopathy (M) Mild (Min) Minimal (Mod) Moderate (n) Number (OR) Odds ratio (Sev) Severe (SD) Standard deviation.

(TS) Total sample $(*) 95 \%$ Confidence interval $(\wedge) 19$ paediatric departments and five/six departments of internal medicine. 
latter finding was consistent with Olsen et al. [38] who reported long-term glycaemic control $(\mathrm{p}<0.0001)$ and diabetes duration before and after puberty onset $(\mathrm{p}<$ 0.05 and $\mathrm{p}<0.001$, respectively) as significantly associated with the development of retinopathy (Table 1). Other findings were generated from samples inclusive of but not specific to the target population.

\section{Prevalence and prediction of nephropathy}

Prevalence of diabetic nephropathy was reported in eight papers [32-35,37-40]. Raile et al. [39] reported data from 262 centres and, as previously reported, Salardi et al. [40] from eleven centres and both Olsen et al. [37] and Olsen et al. [38], from 19 paediatric departments (both) and five/six departments of internal medicine; the number of centres from which data were obtained was unclear in James et al. [34]. In all of these five papers renal function indices employed were not in accordance with current best-practice guideline recommendations. These were however utilised by Broe et al. [32] who reported $18(10.5 \%)$ and 17 (14.8\%) participants with albuminuria, and Garg et al. [33] who reported data from a single eye/kidney clinic. In a study involving 150 participants, 24 (16\%) were reported with albumin excretion indicative of microalbuminuria and eleven (7.3\%) with values indicative of macroalbuminuria. Prevalence data were also reported for 121 participants by Kullberg et al. [35]. For this study, neither number of study sites nor detail of study assessment methods for nephropathy were supplied. At recruitment for fundus photography sample ages ranged mean (SD) 12.4 (2.1) - 41.7 (2.4) years, with subgroups A3 aged 21.9 (2.2) years and A4 27.2 (2.3) years. In these subgroups $14 \%$ and $13 \%$ were reported with urinary albumin excretion greater than $20 \mathrm{mg} / \mathrm{L}$. Factors predicting development of nephropathy were not reported by either Broe et al. [32] or Garg et al. [33].

\section{Prevalence and prediction of hypertension}

The prevalence of hypertension was reported in five papers [33-35,40,41]. Schwab et al. [41] and Salardi et al. [40] reported data derived from 195 and eleven centres, respectively, but did not detail young adult cohort numbers. As previously noted, Kullberg et al. [35] reported prevalence data from 121 eligible participants but did not detail numbers of sites.

Criteria for hypertension in adults with diabetes were revised down to $130 / 80 \mathrm{mmHg}$ earlier this century. All three papers reported the prevalence of hypertension either without stating diagnostic criteria or using what are now out-dated criteria $(140 / 90 \mathrm{mmHg})$. Kullberg et al. [35] and Salardi et al. [40] reported hypertension by their definitions as occurring in $0 \%-9 \%$ of participants. Schwab et al. [41] reported raised systolic and diastolic blood pressures in $11 \%$ and $2.6 \%$ of applicable participants, respectively, with $4.8 \%$ receiving pharmacotherapy. Out-dated criteria were also used by Garg et al. [33] who reported blood pressure values categorised by participants' albumin excretion rate grouping. They reported $34 \%-72.5 \%$ of systolic and $37.7 \%-64.9 \%$ of diastolic ambulatory blood pressure measurements (mean of 24-hour collections) as above the $90 \%$ percentile of normal for age, gender and ethnic group. For participants with macroalbuminuria, over $60 \%$ of day and night-time systolic and diastolic measurements were above the 90th percentile of normal values.

Indices employed by James et al. [34] were in accordance with current best-practice guideline recommendations. Blood pressure measurements were documented in 313 and 306 of participants, with $33.9 \%$ and $30.7 \%$ having mean values within hypertensive ranges, respectively. With anti-hypertensive medication prescribed for $10.2 \%$ of participants a total of 201 (48.4\%) were classified as hypertensive; at least one documented hypertensive measurement was reported in 35 (48.6\%) cohort members prescribed anti-hypertensive medication, across the study period. Participants were more likely to have hypertension if they had no (rather than any) health service contact (OR $0.21,95 \%$ CI $0.1-0.51, \mathrm{p}=0.001$ ) or a longer diabetes duration (each year, OR 1.05, 95\% CI $1.01-1.09, \mathrm{p}=0.006)$. This was in addition to use of continuous subcutaneous insulin infusion therapy (OR $1.8,95 \%$ CI $1.2-2.7, \mathrm{p}=0.004$ ) although this latter finding may have been affected by missing data.

\section{Discussion}

This systematic literature review indicated that vascular complications are common amongst young adults with type 1 diabetes although the results reported varied somewhat. Some form of retinopathy occurred in up to almost half of participants; more severe forms affected up to one in ten. One in six was reported with microalbuminuria; one in 14 had macroalbuminuria. Hypertension occurred in almost one in two participants. In outdated high thresholds this decreased to approximately one in ten participants. The frequency of these complications is concerning since they are largely preventable, are occurring alongside an increasing incidence of type 1 diabetes worldwide, and incur high costs in financial and health-related quality of life terms. $\mathrm{Ng}$ and Morlet [45] flagged the high prevalence and cost of diabetic retinopathy amongst Australians, but failed to differentiate the particular problems of younger onset and hence greater lifetime burden for those with type 1 diabetes. The DiabCo\$t Australia study [46] estimated the minimum annual cost of type 1 diabetes in Australia at between $\$ 430$ to $\$ 570$ million in 2008 , with expenditure increasing with the presence of complications. Real costs were 
acknowledged as higher, as costs associated with disability and premature mortality were not considered.

Identified prevalence rates of retinopathy in this young adult population were elevated compared to recent data for adolescents with type 1 diabetes. Downie et al. [47] reported a prevalence of $12 \%$ between 2005-2009, compared to up to $40 \%$ and $57.6 \%$ in the literature reviewed here $[38,40]$. The review rate was not dissimilar to rates provided for older cohorts of people with type 1 diabetes (within a decade outside the review age criteria). Karadeniz and Yilmaz [48], Esteves et al. [49] and Roy [50] reported retinopathy prevalence of $33.2 \%, 44.4 \%$ and $63.9 \%$, respectively; discrepancies perhaps reflected the trend of increasing prevalence of complications with increasing diabetes duration and age.

In studies where data were obtained using current best practice recommendations, prediction of development of nephropathy was not reported for the young adult age group. Studies of older cohorts of people with type 1 diabetes found diabetic nephropathy associated with indices of diabetes duration and control (increasing $\mathrm{HbA1c}$ ), and with prevalence and severity of other forms of vascular disease and population-wide markers of vascular risk such as triglyceride levels and weight [51-55].

The identified prevalence rates of hypertension in this young adult population were also elevated compared to a study involving a slightly older cohort (mean (SD) age 33.8 (11.8) years at baseline), which reported an increase in elevated systolic and diastolic blood pressures over time. In 2003-2004, 17.9\% and 6\%, respectively, were affected, whereas by 2006-2007 this had increased to $28.8 \%$ and $8.2 \%$. The proportion of participants prescribed antihypertensive medication also increased significantly during this period, from $20.7 \%$ to $34.2 \%$ [56]. However, in another similarly older cohort (mean (SD) age 37 (9) years) only $48 \%$ of those diagnosed and treated for hypertension achieved target values [57], indicating little room for complacency. This is consistent with review findings.

The paucity of blood pressure data for young adults and the indication of poor achievement of treatment goals are particular concerns. Hypertension predisposes to stroke, myocardial infarction, cardiac failure and limb amputation as well as other vascular disease manifestations such as retinopathy and nephropathy. A trend seen in slightly older young adults with type 1 diabetes was of any one end-organ manifestation of vascular disease indicating an increased likelihood of concurrent vascular disease in other areas. For example, in cohorts with mild/severe renal failure, $71.4 \%$ and $83.3 \%$, respectively, also had hypertension [58]. Early detection and prompt treatment are therefore essential, with general population studies clearly demonstrating early diagnosis and adherence to treatment prevents or delays development and progression of end-organ damage [59].
Adherence to sometimes complex, always life-long medication schedules is challenging. 'Typical' versus 'ideal' medication adherence in patients with hypertension has demonstrated nearly double the relative risk of myocardial infarction, angina and stroke [60]. However, Hill et al. [61] cited achievement of up to $80 \%$ adherence rates in routine care and this is especially important for this patient group as cardiovascular disease occurs more than ten times more frequently in those with type 1 diabetes than in age-matched nondiabetes populations [62]. Lack of data on the prevalence of hypertension may hamper prioritisation and appropriate targeting of therapy; important opportunities for treatment may be missed.

Effective prevention interventions rely on identifying modifiable predictors of vascular complications. Data relating specifically to the target population were scarce and this quantitative epidemiological systematic review found glycaemic control as predictive of vascular disease in young adults with type 1 diabetes. Diabetes duration was also flagged, of concern because it is not modifiable and almost half of those who develop the disease do so before age 15 years, many in infancy and childhood [63]. After only nine years with type 1 diabetes almost half of young adults had retinal damage [36] - and probably other vascular disease as well.

On the other hand glycaemic control is modifiable and influential. The deterioration that accrues with disease duration may be ameliorated by better glycaemic control $[9,64]$, with better control being achieved by those who maintain contact and relationships with their diabetes healthcare teams [65]. This flags the crucial importance of ensuring that services are able to support young adults with type 1 diabetes, particularly during the vulnerable period when they leave the paediatric services that supported them as children, establish relationships with adult services and independent self-management practices. It reinforces the importance of regular screening using best practice methods as this offers the best chance for early detection and initiation of appropriate treatment, and consequently to minimise visual loss and blindness, renal failure and dialysis, heart failure and strokes occurring in young adults.

Good quality data are required from adequately powered studies to inform service development, to help nurses and other healthcare professionals risk-stratify and provide appropriate support to young adults with type 1 diabetes, to minimise and defer onset of vascular complications. In most developed countries the data required for high-powered studies are collected routinely by diabetes services. That these data have not been accessed and used to develop algorithms to stratify risk for these young adults is indicative of the lack of priority accorded this problem. 
Some limitations apply to the current review. A search for grey literature such as conference abstracts was not undertaken; neither were experts in this field contacted for unpublished data, nor authors for data from agespecific subsets where these data did not appear in publications. Identification of a specific age range to designate 'young adults' was challenging; we opted to focus on those who would have transitioned out of paediatric into adult care, but use of wider age ranges may have yielded additional data.

Caution also needs to be exercised when considering how review findings can be generalised to the target population of young adults with type 1 diabetes as few studies focused solely on representative samples of this specific age group or involved rural populations. Other omissions were the paucity of studies undertaken in developing countries, and limited data indicating participants' ethnicity. Finally, although studies reporting data collected pre 1993 were excluded in light of the definitive Diabetes Control and Complications Trial [9], it may have taken a number of years for these research findings to change practice such that glycaemic control became central in every-day management. Earlier literature reviewed may therefore be poorly representative of current practice and not reflect prevalence of vascular complications in today's young adults. New primary research is required.

\section{Conclusion}

This is the first systematic review of the prevalence and predictors of retinopathy, nephropathy and hypertension in young adults with type 1 diabetes. While data were limited, underlying vascular disease manifesting as retinopathy and hypertension was common amongst this group, with development predicted by glycaemic control - and probably diabetes duration. With only one of these two factors amenable to clinical management, findings have implications for clinicians, policy-makers, patients and families: to raise the priority of improving glycaemic control as a means to defer and avoid development of complications which otherwise appear near-inevitable.

Clinical messages of this review are the importance of prevention of loss to follow up and provision of appropriate support, particularly around the vulnerable transition period from paediatric to adult-based care. This would ensure support for optimal glycaemic control and enable regular complication screening to be implemented - essential for early detection and treatment in this age group. Quality data are required to be available to clinicians and patients to stratify risk and guide treatment planning, and to inform service development. The message for policy-makers is that the prevalence rates identified make good preventive care essential. The challenge is to make this a realistic option and available to all young adults with type 1 diabetes.

\section{Additional files}

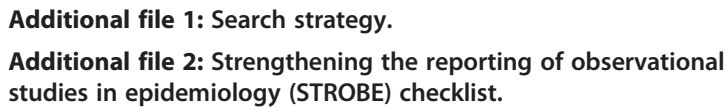

Additional file 3: Summary of extracted information from included literature.

\section{Competing interests}

The authors declare that they have no competing interests.

\section{Authors' contributions}

Paper drafted, revised and agreed by all authors.

\section{Author information}

SJ - RN, CDE, Doctor of Philosophy (Nursing) student, Faculty of Health, University of Technology, Sydney. RG - PhD, MN, RN, Professor of Nursing, Charles Perkins Centrel Sydney Nursing School, University of Sydney. JD - PhD, BAg, Research Assistant, University of Newcastle. LP - PhD, MSc, RN, Professor of Nursing Research, and Practice Development, Faculty of Health, University of Technology, Sydney and Prince of Wales Hospital/Sydney and Sydney Eye Hospitals.

\section{Acknowledgements}

None.

\section{Author details}

${ }^{1}$ Huntsville District Memorial Hospital, Muskoka Algonquin Healthcare, 100 Frank Miller Drive, Huntsville, Ontario P1H 1H7, Canada. ${ }^{2}$ Charles Perkins Centre/Sydney Nursing School, University of Sydney, Sydney, New South Wales 2006, Australia. ${ }^{3}$ University of Newcastle, University Drive, Callaghan, New South Wales 2308, Australia. ${ }^{4}$ Faculty of Health, University of

Technology, Sydney, 15 Broadway, Ultimo, New South Wales 2007, Australia.

Received: 9 January 2014 Accepted: 4 August 2014

Published: 2 September 2014

\section{References}

1. International Diabetes Federation: IDF Diabetes Atlas. Fifthth edition. Brussels: International Diabetes Federation; 2011

2. Catanzariti L, Faulks K, Moon L, Waters A, Flack J, Craig M: Australia's national trends in the incidence of Type 1 diabetes in 0-14 year olds, 2000-2006. Diabet Med 2009, 26(6):596-601.

3. Asao K, Sarti C, Forsen T, Hyttinen V, Nishimura R, Matsushima M: Long-term mortality in nationwide cohorts of children-onset type 1 diabetes in Japan and Finland. Diabetes Care 2003, 26:2037-2042.

4. Orchard T, Forrest K, Kuller L: Lipid and blood pressure treatment goals for type 1 diabetes: 10-year incidence data from the Pittsburgh Epidemiology of Diabetes Complications Study. Diabetes Care 2001, 24:1053-1059.

5. Soedamah-Muthu S, Fuller J, Mulnier H, Raleigh V, Lawrenson R, Colhoun H: All-cause mortality rates in patients with type 1 diabetes mellitus compared with a non-diabetic population from the UK general practice research database, 1992-1999. Diabetologia 2006, 49:660-666.

6. Feltbower RG, Bodansky HJ, Patterson CC, Parslow RC, Stephenson CR, Reynolds C, McKinney PA: Acute complications and drug misuse are important causes of death for children and young adults with type 1 diabetes. Results from the Yorkshire register of diabetes in children and young adults. Diabetes Care 2008, 31:922-926.

7. Shen G: Lipid disorders in diabetes mellitus and current management. Curr Pharm Anal 2007, 3:17-24.

8. Goldberg RB: Cardiovascular disease in patients who have diabetes. Cardiol Clin 2003, 21:399-413. vii.

9. The Diabetes Control and Complications Trial Research Group: The effect of intensive treatment of diabetes on the development and progression of long-term complications in insulin-dependent diabetes mellitus. $N$ Engl J Med 1993, 329:977-986.

10. Marshall S, Flyvbjerg A: Prevention and early detection of vascular complications of diabetes. Br Med J 2006, 333:475-480. 
11. Blum R, Garell D, Hodgman C, Slap G: Transition from child-centered to adult health care systems for adolescents with chronic conditions: a Position Paper of the Society for Adolescent Medicine. J Adolesc Health 1993, 14:570-576.

12. Perry L, Lowe J, Steinbeck K, Dunbabin J: Services doing the best they can service experiences of young adults with type 1 diabetes mellitus in rural Australia. J Clin Nurs 2012, 21(13-14):1955-1963.

13. Hatherly K, Smith L, Overland J, Johnston C, Brown-Singh L: Application of Australian clinical practice management guidelines: the current state of play in a sample of young people living with Type 1 diabetes in the state of New South Wales and the Australian Capital Territory. Diabetes Res Clin Pract 2011, 93(3):379-384.

14. Perry L, Steinbeck K, Dunbabin J, Lowe J: Lost in transition? Access to and uptake of adult health services and outcomes for young people with type 1 diabetes in regional New South Wales. Med J Aust 2010, 193(8):444-449.

15. Holmes-Walker D, Llewellyn A, Farrell K: A transition care programme which improves diabetes control and reduces hospital admission rates in young adults with Type 1 diabetes aged 15-25 years. Diabet Med 2007, 24:764-769.

16. Rapley P, Davidson P: Enough of the problem: a review of time for health care transition solutions for young adults with a chronic illness. J Clin Nurs 2010, 19:313-323.

17. Van Walleghem N, MacDonald C, Dean H: Evaluation of a systems navigator model for transition from pediatric to adult care for young adults with type 1 diabetes. Diabetes Care 2008, 31:1529-1530.

18. Renders C, Valk G, de Sonnaville J: Quality of care for patients with type 2 diabetes mellitus: a long-term comparison of two quality improvements programmes in the Netherlands. Diabet Med 2003, 20:846-852.

19. Shojania K, Ranjii S, McDonald K: Effects of quality improvement strategies for type 2 diabetes on glycemic control: a meta regression analysis. JAMA 2006, 296:427-440

20. Van Dam H, Van der Horst F, Van den Bone B: Provider-patient interaction in diabetes care: effects on patient self-care and outcomes. A systematic review. Patient Educ Couns 2003, 51:17-28.

21. Centre for Reviews and Dissemination: Systematic Reviews. CRD's guidance for undertaking reviews in health care. In Centre for Reviews and Dissemination; 2008.

22. Moher D, Liberati A, Tetzlaff J, DG A: Preferred reporting items for systematic reviews and meta-analyses: the PRISMA statement. PLoS Med 2009, 6(7):1-6.

23. American Diabetes Association: Standards of Medical Care in Diabetes - 2013. Diabetes Care 2013, 36(1):s11-s66.

24. Canadian Diabetes Association Clinical Practice Guidelines Expert Committee: Canadian diabetes association 2013 clinical practice guidelines for the prevention and management of diabetes in Canada. Can J Diabetes 2013, 37(Supp 1):s1-s212.

25. National Institute for Health and Clinical Excellence: Clinical Guideline 15. Type 1 Diabetes: Diagnosis and Management of Type 1 Diabetes in Children, Young People and Adults. London: National Institute for Health and Clinical Excellence; 2004

26. Early Treatment Diabetic Retinopathy Study Research Group: Photocoagulation for diabetic macular edema. Early treatment diabetic retinopathy study report number 1. Arch Opthalmology 1985, 103:1796-1806.

27. Feris FL III: How effective are treatments for diabetic retinopathy? JAMA 1993, 269:1290-1291.

28. Murthy K, Murthy P, Kapur A, Owens D: Mobile diabetes eye care: experience in developing countries. Diabetes Res Clin Pract 2012, 97:343-349.

29. Sano H, Nishimura R, Asao K, Matsudaira T, Morimoto A, Agata T, Sjhimizu H, Tajima N: Blindness and laser photocoagulation in patients with childhood-onset type 1 diabetes in Japan. $\mathrm{Br} J$ Ophthalmol 2009, 93:726-730.

30. Kidney Disease Outcomes Quality Institute: K/DOQI clinical practice guidelines for chonic kidney disease: evaluation,classification, and stratification. Am J Kidney Dis 2002, 39(2 (Suppl 1)):s1-s266.

31. Arfken C, Reno P, Santiago J, Klein R: Development of proliferative diabetic retinopathy in African-Americans and whites with type 1 diabetes. Diabetes Care 1998, 21(5):792-795.

32. Broe R, Rasmussen M, Frydkjaer-Olsen U, Olsen B, Mortensen H, Peto T, Grauslund J: The 16-year incidence, progression and regression of diabetic retinopathy in a young population-based Danish cohort with type 1 diabetes mellitus: the Danish cohort of pediatric diabetes 1987 (DCPD1987). Acta Diabetol 2014, 51:413-420.

33. Garg S, Chase P, Icaza G, Rothman R, Osberg I, Cavmain J: 24-hour ambulatory blood pressure and renal disease ih young subjects with type 1 diabetes. J Diabetes Complications 1997, 11:263-267.

34. James S, Perry L, Gallagher R, Lowe J, Dunbabin J, McElduff P, Acharya S, Steinbeck K: Service usage and vascular complications in young adults with type 1 diabetes. BMC Endocr Disord 2014, 14(39):1-9.

35. Kullberg C, Abrahamsson M, Arnqvist H, Finnström K, Ludvigsson J: Prevalence of retinopathy differs with age at onset of diabetes in a population of patients with Type 1 diabetes. Diabet Med 2002, 19:924-931.

36. LeCaire T, Palta M, Zhang H, Allen C, Klein R, D'Alessio D: Lower-thanexpected prevalence and severity of retinopathy in an incident cohort followed during the First 4-14 years of type 1 diabetes. The Wisconsin Diabetes Registry Study. Am J Epidemiol 2006, 164:143-150.

37. Olsen B, Johannesen J, Sjolie A, Borch-Johnsen K, Hougaard P, Thorsteinsson B, Prammings S, Marinelli K, Mortensen H: Metabolic control and prevalence of microvascular complications in young Danish patients with Type 1 diabetes mellitus. Diabet Med 1999, 16:79-85.

38. Olsen B, Sjolie A, Hougaard P, Johannesen J, Marinelli K, Jacobsen B, Mortensen $\mathrm{H}$ : The significance of the prepubertal diabetes duration for the development of retinopathy and nephropathy in patients with type 1 diabetes. J Diabetes Complications 2004, 18:160-164.

39. Raile K, Galler A, Hofer S, Herbst A, Dunstheimer D, Busch P, Holl R: Diabetic nephropathy in 27,805 children, adolescents, and adults with type 1 diabetes. effect of diabetes duration, A1c, hypertension, dyslipidemia, diabetes onset, and sex. Diabetes Care 2007, 30:2523-2528.

40. Salardi S, Porta M, Maltoni G, Rubbi F, Rovere S, Cerutti F, lafusco D, Tumin S, Calwin V: Infant and toddler type 1 diabetes: complications after 20 years duration. Diabetes Care 2012, 35:829-833.

41. Schwab K, Doerfer J, Hecker W, Grulich-Henn J, Wiemann D, Kordonnouri O, Beyer $P$, Holl R: Spectrum and prevalence of atherogenic risk factors in 27,358 children, adolescents, and young adults with type 1 diabetes. Cross sectional data from the German diabetes documentation and quality management system (DPV). Diabetes Care 2006, 29(2):218-225.

42. Whittemore $\mathrm{R}$, Knafl K: The integrative review: updated methodology. J Adv Nurs 2005, 52(5):546-553.

43. STROBE: Strengthing the reporting of observational studies in epidemiology (STROBE) statement: checklist for cohort, case-control, and cross-sectional studies. 2007, available at www.strobe-statement.org/ fileadmin/Stobe/uploads/checklists/STROBE_checklist_v4_combined.pdf. Accessed 2nd May 2014

44. Schulz K, Altman D, Moher D: CONSORT statement: updated guidelines for reporting parallel group randomisation trials. Ann Intern Med 2010, 152:1-7.

45. $\mathrm{Ng} \mathrm{J}$, Morlet $\mathrm{N}$ : Improving the uptake of screening for diabetic retinopathy. Med J Aust 2013, 198(2):69-70.

46. Colagiuri S, Brnabic A, Gomez M, Fitzgerald B, Buckley A, Colagiuri R: DiabCo \$t Australia Type 1: Assessing the Burden of Type 1 Diabetes in Australia. Canberra: Diabetes Australia; 2009.

47. Downie E, Craig M, Hing S, Cusumano J, Chan A, Donaghue K: Continued reduction in the prevalence of retinopathy in adolescents with type 1 diabetes. Role of insulin therapy and glycemic control. Diabetes Care 2011, 34:2368-2373.

48. Karadeniz Z, Yilmaz M: Duration of diabetes and prevalence of diabetic retinopathy: Istanbul Diabetic Retinopathy Study - IDRS results. Diabetes Metab Syndr 2007, 1:43-48.

49. Esteves J, Kramer C, De Azevedo M, Stolz A, Roggia M, Larangeira A, Miozza S, Rosa C, Humberto Lambert J, Pecis M, Rodrigues T, Henrique Santos Canani L: Prevalence of diabetic retinopathy in patients with type 1 diabetes mellitus. Revista de Associacao Medica Brasileira 2009, 55(3):268-273.

50. Roy M: Diabetic retinopathy in African Americans with type 1 diabetes: the New Jersey 725. 1. Methodology, population, frequency of retinopathy, and visual impairment. Arch Opthalmology 2000, 11(1):97-104.

51. Diamante E: Renal involvement in type 1 (IDDM) diabetes in Spain. Diabetes Res Clin Pract 1997, 38:129-137.

52. Giorgino F, Laviola L, Perin P, Solnica B, Fuller J, Chaturvedi N: Factors associated with progression to macroalbuminuria in microalbuminuric Type 1 diabetic patients: the EURODIAB Prospective Complications Study. Diabetologia 2004, 47:1020-1028.

53. Rodrigues T, Henrique Canani L, Viatroski R, Hoffmann L, Esteves J, Gross J: Masked hypertension, nocturnal blood pressure and retinopathy in 
normotensive patients with type 1 diabetes. Diabetes Res Clin Pract 2010, $87: 240-245$

54. Romero-Aroca P, Fernández-Balart J, Baget-Bernaldiz M, Martinez-Salcedo I, Méndez-Marín I, Salvat-Serra M, Buil-Calvo J: Changes in the diabetic retinopathy epidemiology after 14 years in a population of Type 1 and 2 diabetic patients after the new diabetes mellitus diagnosis criteria and a more strict control of the patients. J Diabetes Complications 2009, 23:229-238.

55. Romero-Aroca P, Sagarra-Alamo R, Baget-Bernaldiz M, Fernandez-Ballart J, Mendez-Marin I: Prevalence and relationship between diabetic retinopathy and nephropathy and its risk factors in the North-East of Spain. A population-based study. Ophthalmic Epidemiol 2010, 17(4):251-265.

56. Wallymahmed W, Morgan C, Gill G, Osman-Hicks V, Chamberlain P, MacFarlane I: Changes in cardiovascular risk factors in a type 1 diabetes population: a 3.5-year study. Pract Diabetes Int 2008, 25(6):223-227.

57. Maahs D, Kinney G, Wadwa P, Snell-Bergeon J, Dabelea D, Hokanson J, Eurlich J, Garg S, Eckel R, Rewers M: Hypertension prevalence, awareness, treatment, and control in an adult type 1 diabetes population and a comparable general population. Diabetes Care 2005, 28(2):301-306.

58. De Pablos-Velasco P, Martinez-Martin F, Aguilar J: Prevalence of hypertension in IDDM patients in the northern Grand Canary Island, according to the WHO/ ISH and JNC-V/ADA criteria. Diabetes Res Clin Pract 1997, 38(13):191-197.

59. U.S. Department of Health and Human Services: The Seventh Report of the Joint National Committee on Prevention, Detection, Evaluation, and Treatment of High Blood Pressure; 2004.

60. Cherry S, Benner J, Hussein M, Tang S, Nichol M: The clinical and economic burden of nonadherence with antihypertensive and lipid-lowering therapy in hypertensive patients. Value Health 2009, 12:489-497.

61. Hill M, Miller N, DeGeest S: ASH position paper: adherence and persistance with taking medication to control high blood pressure. J Clin Hypertens 2010, 12:757-764.

62. Laing S, Swerdlow A, Slater S: Mortality from heart disease in a cohort of 23,000 patients with insulin-treated diabetes. Diabetologia 2003, 46:760-765

63. Craig M, Hattersley A, Donaghue K: Definition, epidemiology and classification of diabetes in children and adolescents. Pediatr Diabetes 2009, 10(Suppl 12):3-12.

64. Klein R, Knudtson M, Lee K, Gangnon R, Klein B: The Wisconsin epidemiologic study of diabetic retinopathy XXII. The twenty-five-year progression of retinopathy in persons with type 1 diabetes. Ophthalmology 2008, 115(11):1859-1868.

65. Dyer P, Lloyd C, Lancashire R, Bain S, Barnett A: Factors associated with clinic non-attendance in adults with type 1 diabetes mellitus. Diabet Med 1998, 15(4):339-343

doi:10.1186/1756-0500-7-593

Cite this article as: James et al:: Prevalence of vascular complications and factors predictive of their development in young adults with type 1 diabetes: systematic literature review. BMC Research Notes 2014 7:593.

\section{Submit your next manuscript to BioMed Central and take full advantage of:}

- Convenient online submission

- Thorough peer review

- No space constraints or color figure charges

- Immediate publication on acceptance

- Inclusion in PubMed, CAS, Scopus and Google Scholar

- Research which is freely available for redistribution

Submit your manuscript at www.biomedcentral.com/submit
Ciomed Central 